\title{
Slippage and Boundary Layer Probed in an Almost Ideal Gas by a Nanomechanical Oscillator
}

\begin{abstract}
M. Defoort, K. J. Lulla, T. Crozes, O. Maillet, O. Bourgeois, and E. Collin
Université Grenoble Alpes, CNRS Institut NÉEL, BP 166, 38042 Grenoble Cedex 9, France

(Received 25 March 2014; revised manuscript received 17 August 2014; published 23 September 2014)

We measure the interaction between ${ }^{4} \mathrm{He}$ gas at $4.2 \mathrm{~K}$ and a high-quality nanoelectromechanical string device for its first three symmetric modes (resonating at 2.2, 6.7, and $11 \mathrm{MHz}$ with quality factor $Q>0.1 \times 10^{6}$ ) over almost 6 orders of magnitude in pressure. This fluid can be viewed as the best experimental implementation of an almost ideal monoatomic and inert gas of which properties are tabulated. The experiment ranges from high pressure where the flow is of laminar Stokes-type presenting slippage down to very low pressures where the flow is molecular. In the molecular regime, when the meanfree path is of the order of the distance between the suspended nanomechanical probe and the bottom of the trench, we resolve for the first time the signature of the boundary (Knudsen) layer onto the measured dissipation. Our results are discussed in the framework of the most recent theories investigating boundary effects in fluids (both analytic approaches and direct simulation Monte Carlo methods).
\end{abstract}

DOI: 10.1103/PhysRevLett.113.136101

Micro- and nanotechnologies have driven advances in various fields taking advantage of electronic, mechanical, and even, nowadays, fluid properties exploited at very small scales (micronic and submicronic) [1]. The so-called area of micro- and nanofluidics is today under intense research, with applications ranging from chemistry to biology [2-4]. Micro- and nanomechanical elements (MEMS and NEMS) are then key tools to probe and interact with gases and liquids, which are often simply air or water [5-7]. Especially with NEMS, the device noninvasivity can be pushed down to the submicrometer scale.

Beyond technological applications and engineering problems, fundamental issues of fluid mechanics are also intimately associated to this research. These are essentially linked to the actual interaction between the fluid and a wall of some kind. In conventional, macroscopic, and viscous fluid flow, the boundary condition that is used to describe the physical phenomenon is the so-called no-slip property: at the level of the obstacle, the fluid is assumed to be clamped on irregularities of the surface and the tangential velocity goes to zero $[8,9]$. This boundary condition becomes completely wrong in rarefied gases $[9,10]$, micronano fluidic devices $[11,12]$ and quantum fluids such as mixtures of liquid ${ }^{3} \mathrm{He}$ and ${ }^{4} \mathrm{He}[13,14]$. A stunning and counterintuitive example of this is water flow in carbon nanotubes demonstrating gigantic slippage [15].

Comprehending what happens physically between a fluid and a solid wall is, thus, essential for both practical applications and our fundamental understanding of fluid dynamics [16,17]. With MEMS and NEMS, this can be studied through oscillating flows in (more or less) confined geometries $[16,18,19]$. In the boundary (or Knudsen) layer of thickness about one mean-free path next to the solid surface, a rarefaction phenomenon occurs because particles
PACS numbers: 68.47.-b, 51.10.+y, 62.25.-g, 81.07.-b

collide more frequently with the wall than among themselves: this leads to a strong deviation of the statistical distribution of velocities from the Maxwellian equilibrium state (reached in the bulk). The flow is non-Newtonian and presents a nonzero tangential velocity (the slip effect) and reduced viscosity. There is today an extensive theoretical literature on the structure of the Knudsen layer, with even some predictions that do not agree with each other [17,20-26]; at the same time, direct experimental evidence of what actually happens within this boundary fluid is lacking.

In this Letter, we report on experiments conducted in ${ }^{4} \mathrm{He}$ gas at $4.2 \mathrm{~K}$ using a nanoelectromechanical device. We measure the friction experienced by the probe immersed in the fluid through one of its mechanical mode's resonance frequency shift and broadening. The three first symmetric modes have been used, and the pressure of the gas has been varied over almost 6 orders of magnitude. In the rarefied gas limit, we measure for the first time a decrease of the damping acting onto the NEMS (with respect to the free molecular expression), which is evidence of the boundary layer effect. Two similar devices have been used with different distances from the suspended element to the bottom trench, thus, proving unambiguously the boundary layer signature.

The NEMS fabrication and design can be found in Ref. [27]. It is excited and detected by means of the magnetomotive scheme [28]. A sinusoidal current $I_{0} \cos (\omega t)=V(t) / 1 \mathrm{k} \Omega$ is fed through the suspended beam via a cold bias resistor, which produces a Laplace force from a reasonably small magnetic field $B$. The out-ofplane motion is detected through the induced emf voltage $U(t)$ with a lock-in amplifier. A careful calibration procedure is used to deduce the applied forces and resulting 


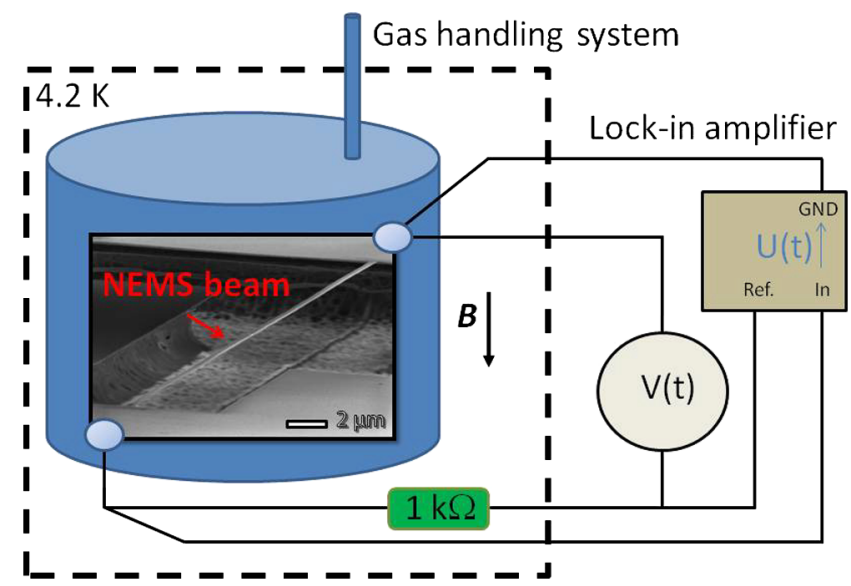

FIG. 1 (color online). Schematic of the experimental setup and SEM picture of the silicon-nitride nanomechanical device. The dimensions are $w=300 \mathrm{~nm}$ wide, $e=100 \mathrm{~nm}$ thick, and $h=100 \mu \mathrm{m}$ long. A thin layer of aluminum $(30 \mathrm{~nm})$ has been evaporated on top to create electrical contacts. The distance between suspended NEMS and bottom of the chip is $g=4 \mu \mathrm{m}$. The whole cell is placed in a coil producing up to $1 \mathrm{~T}$ magnetic fields $B$ in plane with respect to the chip and perpendicular to the NEMS.

velocities [29]. The setup is schematically depicted in Fig. 1, and typical resonance lines are presented in Fig. 2 for the first flexural mode. Each data point has been taken with at least two different excitation levels in order to make sure that heating and nonlinear effects are negligible (see the Supplemental Material [30]). NEMS velocities $U_{0}$ have been kept small enough to ensure that the Reynolds number $R_{e}=\rho_{g} w U_{0} / \eta$ is smaller than 0.2 for all measurements.

When the mean-free path of atoms or molecules is sufficiently short compared to experimental dimensions

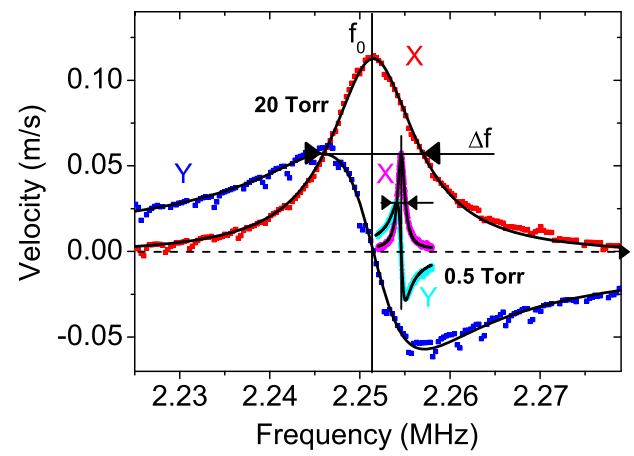

FIG. 2 (color online). Two typical resonance lines (mode No. 1) obtained for pressures above and below the crossover from Navier-Stokes to molecular flow (see Fig. 3). The lock-in amplifier leads to a homodyne detection giving access to the two quadratures $X$ and $Y$ [29]. To keep the signal detectable, both magnetic field and current are increased as the damping increases (see text). Lines are Lorentzian fits giving access to resonance frequency $f_{0}$ and linewidth $\Delta f$. (object width, cavity width), the dynamics can be described by the well-known Navier-Stokes equation $[8,41]$. The first order correction to the dynamics when the mean-free path becomes comparable to the size of the immersed objects is a modification of the boundary condition, introducing a phenomenological slip length $[14,17,20,23,25,26]$. In experiments, this parameter is essentially a fit parameter (see e.g., Refs. [11,12,15,42,43]), which theoretically depends on the nature of the surface interaction (diffusive or specular, see e.g., Refs. [20,44]).

${ }^{4} \mathrm{He}$ gas at $4.2 \mathrm{~K}$ is the best experimental realization of a monoatomic and inert ideal gas. For pressures below $1 \mathrm{bar}$, the gas is classical (mean distance between atoms much larger than the thermal de Broglie wavelength). Thermodynamical properties including the scattering cross sections can be found in the literature [45-48]. Since the beam length $h$ is the largest length scale, we model all friction mechanisms as being local and integrate over the mechanical resonance mode shape to obtain the total damping parameter (see the Supplemental Material for technical details [30]). The interaction force per unit length between fluid and solid is written as

$$
\frac{d F_{g}(\omega, z)}{d z}=+\rho_{g} \omega^{2} S_{l} \Lambda(\omega) \Psi_{n}(z) x_{n}(\omega),
$$

with $\rho_{g}$ the gas mass density, $S_{l}$ the characteristic cross section presented by the beam, and $\Psi_{n}(z) x_{n}(\omega)$ the displacement of the beam element at abscissa $z$ ( $\Psi_{n}$ being the normalized mode shape, $x_{n}$ the overall harmonic motion amplitude for mode $n$, and $\omega=2 \pi f$ the angular frequency). In the high-pressure gas, at low enough NEMS velocities the fluid dynamics is laminar and follows the Navier-Stokes equation $[8,49]$. Following Ref. [50], in the case of a rectangular beam we write for the damping coefficient $\Lambda(\omega)$

$$
\Lambda(\omega)=\Gamma(\omega) \Omega(\omega),
$$

where $\Gamma(\omega)$ is the well-known Stokes' (complex) function [51] and $\Omega(\omega)$ a correction function valid for $e \ll w$ (beam thickness and width, respectively) [50]. Slippage is incorporated with the further renormalization [14,52]

$$
\Lambda(\omega) \rightarrow 1+\frac{1}{(\Lambda(\omega)-1)^{-1}-i\left(\frac{w / 2}{\delta}\right)^{2}\left(\frac{l_{\text {slip }}}{l_{\operatorname{slip}}+w / 2}\right)},
$$

with $\delta=2 \sqrt{\eta / \rho_{g} \omega}$ the viscous penetration depth ( $\eta$ being the dynamic viscosity of the gas) and $l_{\text {slip }}$ the slip length. $l_{\text {slip }}$ can be expressed as a function of the specular fraction $\sigma$ of reflected particles from the probe's surface ( 1 is purely specular and 0 is diffusive) $[14,22,24,44]$

$$
l_{\text {slip }} \approx 1.15\left(\frac{1+\sigma}{1-\sigma}\right) l_{m f p} .
$$




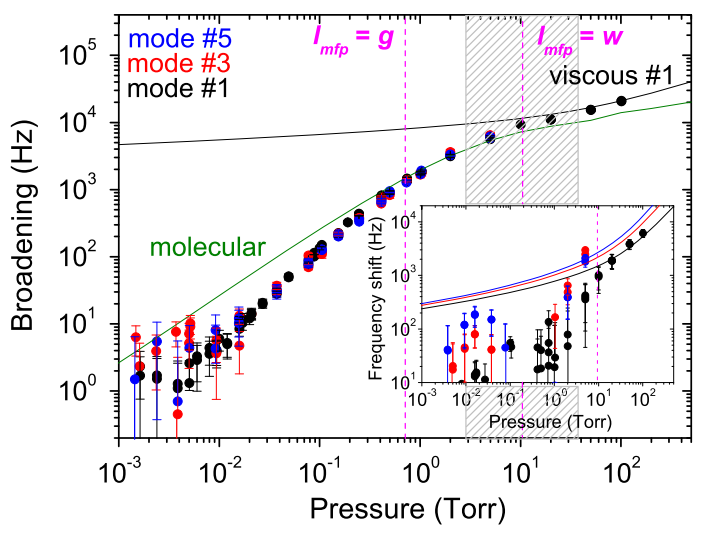

FIG. 3 (color online). Gas friction as measured by the NEMS device. The three colors stand for the first three symmetric modes (1, 3, and 5). Main: damping inferred from the broadening of the linewidth $\Delta f-\Delta f^{\mathrm{vac}}$. The intrinsic contribution $\Delta f^{\mathrm{vac}}$ has been subtracted. The black line is the calculation for the Navier-Stokes limit while the green (light) line corresponds to the molecular expression. Inset: reactive contribution extracted from the frequency shift $f_{0}-f_{0}^{\text {vac }}$. Same conventions as of main graph (plus Navier-Stokes calculations for modes No. 3 and No. 5). The dashed verticals mark the pressures when $l_{m f p} \approx g=4 \mu \mathrm{m}$ and $l_{m f p} \approx w=300 \mathrm{~nm}$, and the shaded zone shows the region where the flow is neither Navier-Stokes nor molecular (see text).

The slip length is proportional to the mean-free path and diverges for perfectly specular conditions on the immersed object. We show in Fig. 3 the calculation based on the model for mode No. 1 with the reasonable value of $\sigma=0.5$. Above $l_{m f p} \approx w$, the curve fits the data rather well for both dissipative and reactive components [computed from imaginary and real parts of $\Lambda(\omega)$, respectively]. For higher modes, unfortunately, the damping was too large and no data could be acquired. Note that there is no other fitting parameter in the model (see the Supplemental Material [30]).

When the mean-free path of atoms or molecules becomes very large compared to the transverse dimensions of the solid body in contact with the gas (container or obstacle), it is said to be in the "molecular flow" (or ballistic) limit [10]. The same concept can be applied to a (classical or quantum) liquid, even though the elementary excitation will not be a bare atom or molecule, but a "quasiparticle" due to the strong interactions of each constitutive particle with its surroundings [44]. For a classical gas, few micro- or nanofluidics experiments have been conducted in the molecular flow limit: usually, the pressure range investigated corresponds to the slip flow described above or the transition flow regime where neither Navier-Stokes nor molecular theories apply (shaded region in Fig. 3) $[12,41,43]$. However, in this pressure range, an elegant similarity theorem has been recently demonstrated in Ref. [16] describing the damping of the gas onto a nanomechanical object.

In the molecular flow limit, the Navier-Stokes equation does not apply and the interaction between the moving body and the gas should be calculated from the transfer of momentum integrated over all particles bouncing off the device $[10,41,53]$. We write

$$
\frac{d F_{g}(\omega, z)}{d z}=+2 \alpha \rho_{g} w \overline{v_{g}}\left[i \omega \Psi_{n}(z) x_{n}(\omega)\right]
$$

with $\overline{v_{g}}$ the average thermal velocity in the gas and $\alpha$ a number close to 1 taking into account details of the scattering process (in particular $\sigma$, see the Supplemental Material [30]); $-i \omega \Psi_{n}(z) x_{n}(\omega)$ is the velocity of beam element $d z$, and the friction presents only a dissipative component. The calculation based on the cross-over expression given in Ref. [41] is shown in Fig. 3. As expected from Eq. (5), the measured damping is independent of frequency in the molecular regime, and the reactive component falls towards zero. Note that the frequency shift due to the mass of ${ }^{4} \mathrm{He}$ adsorbed layers has been subtracted (Supplemental Material [30]), which explains the rather large error bars in the Fig. 3 inset.

Equation (5) predicts a damping proportional to pressure $P$ in the molecular limit. Note that within the simple modeling, the calculation has no free parameter (Supplemental Material [30]). However, in Fig. 3 the measured result is clearly below the calculation (up to an order of magnitude around $10^{-3}$ Torr). The deviation occurs precisely for the pressure where the mean-free path $l_{m f p}$ is of the order of the distance between the NEMS device and the bottom of the chip g, about $4 \mu \mathrm{m}$. To quantitatively analyze this effect, we plot the normalized damping to the ideal molecular expression $2 \rho_{g} w \bar{v}_{g}$ in Fig. 4. At low pressures, the theoretical prediction tends towards the constant $\alpha$, which should be 1 for perfectly

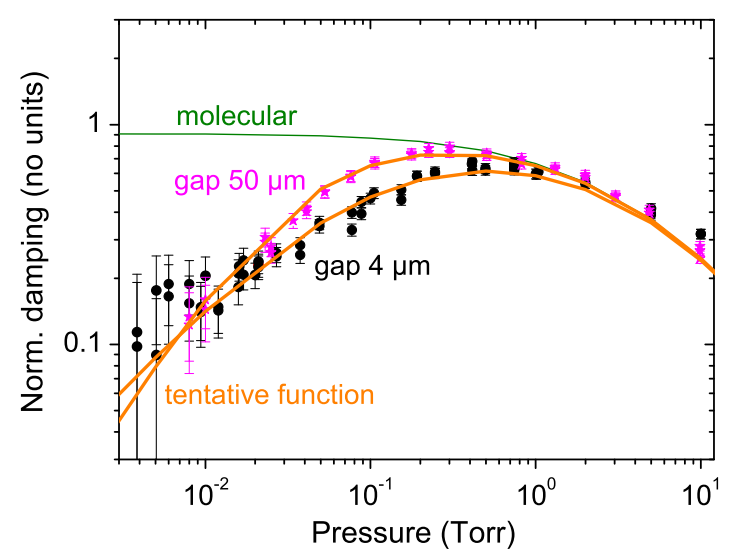

FIG. 4 (color online). Measured dissipation normalized to the ideal calculated molecular result of Eq. (5). The first mode of the device of Fig. 3 is shown (black dots) together with the data of another device having $g=50 \mu \mathrm{m}$ (magenta or light stars). The green (light) line is the normalized green (light) line presented in Fig. 3. Below typically $l_{m f p} \approx g$ the measured friction decreases significantly. The two thick lines are tentative fits discussed in the text, with respectively $a=0.80 \pm 0.1, b=0.18 \pm 0.05$ $(g=4 \mu \mathrm{m})$ and $a=1.15 \pm 0.1, b=0.65 \pm 0.1(50 \mu \mathrm{m})$. 
specular NEMS surfaces (green thin line). To rule out any effects linked to the device itself, the measurements have been conducted at very low velocities and injected powers while the non-Maxwellian effects on Eq. (5) due to the gas-NEMS boundary layer have been estimated: these are all negligible (see Supplemental Material [30]).

The claim is, thus, that when $l_{m f p}>g$, the nanomechanical probe gradually enters into the Knudsen layer attached to the bottom trench, which diminishes the measured viscosity, as expected. In Fig. 4 we also show the normalized data obtained for a similar device having $g=50 \mu \mathrm{m}$. The decrease in the measured damping occurs clearly at lower pressures, which validates the claim. To our knowledge, this effect has never been reported before, while the intense recent theoretical investigations were clearly calling for experimental inputs (see e.g., Ref. [17] and references therein).

Mathematically, the problem at hand is particularly tough, and for most predictions it requires accurate (and demanding) numerical simulations. As a result, the most recent direct simulation Monte Carlo methods (DSMC) $[17,25]$ contradict older findings: in particular the prediction of a reduction of the effective boundary layer viscosity to maximum $1 / 2$ of its bulk value $\eta_{\infty}$ for a perfectly diffusive surface. Our experimental findings seem to contradict this point as well, since in Fig. 4 the measured decrease of the effective viscosity rises up to a factor of 10 at the lowest pressures with no sign of saturation. In this sense, experiments are compatible with the most developed DSMC theories.

The quantitative analysis of the data can be pushed one step further, building again on the theoretical work of Refs. [17,25]. The effective viscosity is supposed to scale as $\eta_{\infty}\left(g / l_{m f p}\right)^{a}$ for large mean-free paths. In order to match high and low pressure limits within the molecular range, we propose the phenomenological expression

$$
\text { damping } \propto P \frac{1}{1+b\left(l_{m f p} / g\right)^{a}} \text {. }
$$

This function is the one used on Fig. 4 with $g$ equal to 4 and $50 \mu \mathrm{m}$ for the two samples, with slightly different $a$ and $b$ fit parameters (see caption). While the fits are quite convincing, the obtained $a$ exponent does not seem to match theoretical predictions, which calls for both new experimental and theoretical investigations.

In conclusion, we have measured the friction experienced by a nanomechanical device immersed in an almost ideal gas, ${ }^{4} \mathrm{He}$ at $4.2 \mathrm{~K}$. The pressure has been ranged from about $10^{-3}$ Torr where the flow is molecular up to about $1 \mathrm{~atm}$ where the gas is described by a laminar NavierStokes flow. The first three symmetric modes of the NEMS structure have been used to analyze the dependence to frequency or mode shape. We inferred that a rather large slippage occurs in the Navier-Stokes limit, consistent with a reasonably large fraction of specular reflections of particles off the probe. At low pressures in the rarefied gas limit, when the mean-free path of atoms exceeds the distance to the bottom of the chip we measured a large deviation with respect to the ideal $\propto P$ damping molecular expression. We interpret the effect as the reduction of the effective viscosity occurring in the boundary layer and try to consistently fit the data with respect to the most recent theoretical DSMC results. We, indeed, reproduce a power-law decrease of the effective viscosity at very low pressures (long mean-free paths), which does not saturate to $1 / 2$ of its bulk value. However, the decrease is much faster than the theoretically expected one. Our findings should help in modeling the structure of the Knudsen boundary layer, which is extremely valuable as far as the comprehensive understanding of rarefied flows in micro- and nanosystems is concerned.

The authors gratefully thank H. Godfrin for extremely valuable discussions and J. Minet and C. Guttin for help in setting up the experiment. One of the authors (E.C.) also wants to acknowledge stimulating discussions with K. Ekinci and J.-P. Poizat. We wish to thank T. Fournier for help in the microfabrication process. We acknowledge the support from MICROKELVIN, the EU FRP7 low temperature infrastructure Grant No. 228464, and the ANR French Grant QNM No. 040401.

*Corresponding author. eddy.collin@neel.cnrs.fr

[1] G. Karniadakis, A. Beskok, and N. Aluru, Microflows and Nanoflows (Springer, New York, 2005).

[2] C.-M. Ho and Y.-C. Tai, Annu. Rev. Fluid Mech. 30, 579 (1998).

[3] T. P. Burg, M. Godin, S. M. Knudsen, W. Shen, G. Carlson, J. S. Foster, K. Babcock, and S. R. Manalis, Nature (London) 446, 1066 (2007).

[4] M. Rosenauer and M. J. Vellekoop, Appl. Phys. Lett. 95, 163702 (2009).

[5] A. Maali, C. Hurth, R. Boisgard, C. Jai, T. Cohen-Bouhacina, and J.-P. Aimé, J. Appl. Phys. 97, 074907 (2005).

[6] J. Dorignac, A. Kalinowski, S. Erramilli, and P. Mohanty, Phys. Rev. Lett. 96, 186105 (2006).

[7] C. I. Bouzigues, P. Tabeling, and L. Bocquet, Phys. Rev. Lett. 101, 114503 (2008).

[8] L. D. Landau and E. M. Lifshitz, Fluid Mechanics, 2nd ed. (Pergamon, New York, 1987).

[9] C.M. Van Atta, Vacuum Science and Engineering (McGraw-Hill, New York, 1965).

[10] G. N. Patterson, Molecular Flow of Gases (John Wiley \& Sons, New York, 1956).

[11] U. Ulmanella and C.-M. Ho, Phys. Fluids 20, 101512 (2008).

[12] A. Siria, A. Drezet, F. Marchi, F. Comin, S. Huant, and J. Chevrier, Phys. Rev. Lett. 102, 254503 (2009).

[13] S. Perisanu and G. Vermeulen, J. Low Temp. Phys. 135, 701706 (2004). 
[14] R. M. Bowley and J. R. Owers-Bradley, J. Low Temp. Phys. 136, 15 (2004).

[15] J. K. Holt, H. G. Park, Y. Wang, M. Stadermann, A. B. Artyukhin, C. P. Grigoropoulos, A. Noy, and O. Bakajin, Science 312, 1034 (2006).

[16] E. C. Bullard, J. Li, C. R. Lilley, P. Mulvaney, M. L. Roukes, and J. E. Sader, Phys. Rev. Lett. 112, 015501 (2014).

[17] C. R. Lilley and J. E. Sader, Proc. R. Soc. A 464, 2015 (2008).

[18] K. L. Ekinci, D. M. Karabacak, and V. Yakhot, Phys. Rev. Lett. 101, 264501 (2008).

[19] C. Lissandrello, V. Yakhot, and K. L. Ekinci, Phys. Rev. Lett. 108, 084501 (2012).

[20] D. Einzel, P. Panzer, and M. Liu, Phys. Rev. Lett. 64, 2269 (1990).

[21] P. A. Thompson and M. O. Robbins, Phys. Rev. A 41, 6830 (1990).

[22] C. E. Siewert, Phys. Fluids 15, 1696 (2003).

[23] D. A. Lockerby, J. M. Reese, D. R. Emerson, and R. W. Barber, Phys. Rev. E 70, 017303 (2004).

[24] M. Fichman and G. Hetsroni, Phys. Fluids 17, 123102 (2005).

[25] C. R. Lilley and J. E. Sader, Phys. Rev. E 76, 026315 (2007).

[26] S. H. Kim, H. Pitsch, and I. D. Boyd, Phys. Rev. E 77, 026704 (2008).

[27] M. Defoort, K. J. Lulla, C. Blanc, H. Ftouni, O. Bourgeois, and E. Collin, J. Low Temp. Phys. 171, 731 (2013).

[28] A. N. Cleland and M. L. Roukes, Sens. Actuators 72, 256 (1999).

[29] E. Collin, M. Defoort, K. Lulla, T. Moutonet, J.-S. Heron, O. Bourgeois, Yu. M. Bunkov, and H. Godfrin, Rev. Sci. Instrum. 83, 045005 (2012).

[30] See the Supplemental Material at http://link.aps.org/ supplemental/10.1103/PhysRevLett.113.136101, which includes Refs. [31-40], for technical details.

[31] V. G. Chernyak, B. T. Porodnov, and P. E. Suetin, J. Eng. Phys. Thermophys. 26, 309 (1974).

[32] P. H. Schildberg, Ph.D. thesis, 1988; reprinted in H. Godfrin and R. E. Rapp, Adv. Phys. 44, 113 (1995).

[33] J. G. Dash, Films on Solid Surfaces (Academic Press, London, 1975).
[34] G. F. Cerofolini, J. Low Temp. Phys. 6, 473 (1972).

[35] G. J. Goellner, J. G. Daunt, and E. Lerner, J. Low Temp. Phys. 21, 347 (1975); A. Novaco, J. Low Temp. Phys. 21, 359 (1975).

[36] P. M. Morse and K. U. Ingard, Theoretical Acoustics, 1st ed. (Princeton University Press, Princeton, NJ, 1986).

[37] C. A. Van Eysden and J. E. Sader, J. Appl. Phys. 101, 044908 (2007); C. P. Green and J. E. Sader, J. Appl. Phys. 92, 6262 (2002).

[38] J. M. Ziman, Electrons and Phonons (Oxford University Press, New York, 2001).

[39] M. Sinvani, M. W. Cole, and D. L. Goodstein, Phys. Rev. Lett. 51, 188 (1983).

[40] P. Taborek and L. J. Senator, Phys. Rev. Lett. 56, 628 (1986).

[41] R. B. Bhiladvala and Z. J. Wang, Phys. Rev. E 69, 036307 (2004).

[42] C. I. Bouzigues, P. Tabeling, and L. Bocquet, Phys. Rev. Lett. 101, 114503 (2008).

[43] J. Laurent, A. Drezet, H. Sellier, J. Chevrier, and S. Huant, Phys. Rev. Lett. 107, 164501 (2011).

[44] H. Hojgaard Jensen, H. Smith, P. Wölfle, K. Nagai, and T. Maack Bisgaard, J. Low Temp. Phys. 41, 473 (1980).

[45] V. D. Arp and R. D. McCarty, Thermophysical Properties of Helium-4 from 0.8 to $1500 \mathrm{~K}$ with Pressures to $2000 \mathrm{MPa}$, NIST Technical Note 1334, NIST, Boulder, 1989.

[46] J. A. Gracki, G. P. Flynn, and J. Ross, Technical Report No. MIT-50-PU, 1969.

[47] R.C Reid, J. M. Prausnitz, and B. E. Poling, The Properties of Gases and Liquids, 4th ed. (McGraw-Hill, New York, 1987).

[48] E. Bich, J. Millat, and E. Vogel, J. Phys. Chem. Ref. Data 19, 1289 (1990).

[49] E. Collin, L. Filleau, T. Fournier, Y. M. Bunkov, and H. Godfrin, J. Low Temp. Phys. 150, 739 (2008).

[50] J. E. Sader, J. Appl. Phys. 84, 64 (1998).

[51] G. G. Stokes, Mathematical and Physical Papers (Cambridge University Press, London, 1901), Vol. 3 p. 38.

[52] D. C. Carless, H. E. Hall, and J. R. Hook, J. Low Temp. Phys. 50, 583 (1983).

[53] K. Yamamoto and K. Sera, Phys. Fluids 28, 1286 (1985). 